\section{Pontine infarct during deep brain stimulation surgery as the cause of postoperative hemiparesis}

\section{Zulfiqar Ali, Hemanshu Prabhakar', Navid Wani ${ }^{2}$}

Sir,

Deep brain stimulation (DBS) of subthalamic nucleus and globus pallidus is increasingly being used for treatment of Parkinson's disease (PD) and other movement disorders. The procedure involves the stereotaxic placement of an implantable electrode with four active contacts within the target. This is followed by tunnelling of the external cables of the electrodes, which are connected to an externally implanted pulse generator. A wide array of complications can occur during this procedure. Complications reported in literature are related to the device, the procedure, the electrical stimulation and the spread of current. ${ }^{[1,2]}$

Of particular concern to the anaesthesiologists are the perioperative complications; some of them, in our experience, were unusual. We report a case where a patient undergoing DBS for PD developed altered sensorium intraoperatively as a result of a lacunar infarct in the pons.

A 60-year-old male patient, weighing $62 \mathrm{~kg}$ was scheduled for DBS procedure. His past medical history was unremarkable. The first part of the procedure, that is, electrode placement, was done under monitored anaesthesia care. In the operation theatre, routine monitoring included 5-lead electrocardiogram, non-invasive blood pressure, pulse oximetry and respiratory rate. Supplemental oxygen was administered via nasal prongs at a flow rate of 2 litres per minute. The patient was positioned supine on the operating table in a stereotactic frame with $10^{\circ}$ head up tilt. Following placement of electrodes on the left side, as the electrodes were being placed on the right side, the patient was suddenly uncooperative. He stopped following commands from the neurologist and became restless. The procedure was abandoned and patient was shifted for computed tomography (CT) of head. The scan revealed a large pneumocephalus but no operative site haematoma or any other abnormality [Figure 1a].

On the second postoperative day, the patient developed hemiparesis on the left side. Meanwhile, his sensorium improved and he started following commands. A repeat $\mathrm{CT}$ scan was performed which now revealed right midbrain small lacunar infarct and reduced pneumocephalus [Figure 1b]. Later on, the patient recovered in the intensive care unit.

The various adverse effects of DBS related to the surgical procedure, the implanted device and electrical stimulation of areas adjacent to the target have been described in literature. ${ }^{[3]}$ Some of these include intracerebral haematoma, ischaemic stroke, seizures, lead migration, erosion and breakage, dyskinesias, dysarthria, mood changes, Horner's syndrome, etc., Of particular concern to the anaesthesiologists are the perioperative complications. Altered sensorium as a result of pneumocephalus is a known entity. ${ }^{[4]}$ The clinical pattern depends on the territory involved in the pons. Pontine infarcts are known to produce a variegated clinical picture ranging from sensory to motor symptoms.
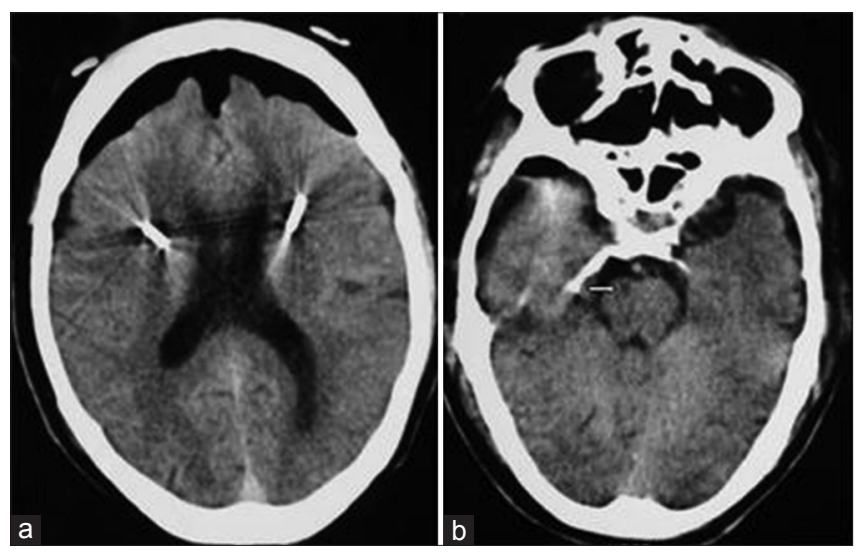

Figure 1: (a) A non-contrast computed tomographic scan of head showing large bifrontal haematoma and artifacts due to electrodes, (b) A right midbrain small lacunar infarct (white arrow)

Department of Neuroanaesthesiology, Sheri Kashmir Institute of Medical Sciences, Srinagar, ${ }^{2}$ Vardhman Mahavir Medical College and Safdarjung Hospital, New Delhi, 'Department of Neuroanaesthesiology, Neurosciences Centre, All India Institute of Medical Sciences, New Delhi, India 
Features like dysarthria, ataxia, vertigo, dizziness, tetra paresis, pseudobulbar palsy, etc., may be seen..$^{[5]}$ Our case illustrates altered mental status coinciding with the developing pneumocephalus and infarction in the pons.

\section{REFERENCES}

1. Beric A, Kelly PJ, Rezai A, Sterio D, Mogilner A, Zonenshayn M, et al. Complications of deep brain stimulation surgery. Stereotact Funct Neurosurg 2001;77:73-8.

2. Venkatraghavan L, Manninen P, Mak P, Lukitto K, Hodaie M, Lozano A. Anesthesia for functional neurosurgery: Review of complications. J Neurosurg Anesthesiol 2006;18:64-7.

3. Valldeoriola F, Pilleri $\mathrm{M}$, Tolosa $\mathrm{E}$, Molinuevo JL, Rumià $\mathrm{J}$, Ferrer E. Bilateral subthalamic stimulation monotherapy in advanced Parkinson's disease: Long term follow-up of patients. Mov Disord 2002;17:125-32.
4. Jain V, Prabhakar H, Rath GP, Sharma D. Tension pneumocephalus following deep brain stimulation surgery with bispectral index monitoring. Eur J Anaesthesiol 2007;24:203-4.

5. Kumral E, Bayülkem G, Evyapan D. Clinical spectrum of pontine infarction. Clinical-MRI correlations. J Neurol 2002;249:1659-70.

\begin{tabular}{|l|l|}
\hline \multicolumn{2}{|c|}{ Access this article online } \\
\hline Quick Response Code: & Website: \\
\hline & www.jnaccjournal.org \\
\cline { 2 - 2 } & \\
\hline
\end{tabular}

